\title{
Direct-to-consumer pharmacogenomic testing assessed in a US-based study
}

\author{
AK Daly \\ Professor of Pharmacogenetics, Institute of Cellular Medicine, Newcastle University, Newcastle upon Tyne, UK
}

TITLE Direct-to-consumer pharmacogenomic testing is associated with increased physician utilisation

AUTHORS Bloss CS, Schork NJ, Topol EJ

JOURNAL J Med Genet 20I4: 5I: 83-89.

DECLARATION OF INTERESTS No conflict of interest declared.

\author{
Correspondence to AK Daly \\ Institute of Cellular Medicine \\ Newcastle University \\ William Leech Building \\ Medical School \\ Framlington Place \\ Newcastle upon Tyne NE2 $4 \mathrm{HH}$ \\ UK
}

e-mail a.k.daly@ncl.ac.uk

\section{SUMMARY}

Direct-to-consumer genetic testing has been around for approximately 20 years. Pharmacogenomic testing is a specialised form of this testing which informs the risk of developing particular toxicities or showing a lack of response when prescribed certain drugs. These tests were widely available in the US via companies such as 23andMe' but, somewhat controversially, public access has been recently restricted by the US Food and Drug Administration (FDA). ${ }^{2}$ More limited, but similar, testing is also available in the UK, though consumers are expected to speak to a 'genetics trained' advisor first.

Bloss and colleagues conducted a study on participants in the Scripps Genomic Health Initiative (SGHI) involving more than 3,000 individuals from academic institutes and technology companies in California. The participants were offered subsidised commercial pharmacogenomic testing. The current study compares certain outcomes between participants who had received the results of pharmacogenomic testing (approximately 35\% of 1325 study participants) and those who were still awaiting their results (approximately 65\%). The outcomes compared included concerns about privacy, whether results were shared with family members and doctors and whether the participants availed themselves of further free genetic counselling. The group that had received results were slightly more likely to have visited their primary care physician and significantly more likely to plan to share their results with their doctor than the group still awaiting the results.After receiving the results, participants were also more likely to decide to seek genetic counselling.
The 475 participants who had already received their results were subdivided into those at high (4I participants) and low (434 participants) pharmacogenomic risk, based on data they provided about medicines taken currently or previously. These groups were compared on a number of outcomes such as visiting their primary care physician or another healthcare provider and level of distress after receiving their results. The main findings were that those in the high risk group were considerably more likely to have visited their primary care physician after receiving the results and were slightly more likely than the low risk group to share their results with their doctor. They were also twice as likely as the low risk group to seek genetic counselling.

Levels of satisfaction with the process, including finding the test results useful, understanding them and sharing results with both their family and their doctor, were high for participants who had received results. In particular, the group with test results was significantly more likely to consider the results useful and understand them compared with the expectations of the group still awaiting the findings. There was no evidence for any changes in psychological health or increased distress following release of results.

\section{CLINICAL OPINION}

The study provides reassurance that consumers who order pharmacogenomic tests do not suffer harm and seem able to deal sensibly with the information provided. It raises the important question of whether additional health care resources might be needed to cope with 
increased demand for medical advice following such tests. The study also has a number of limitations, for example study participants were not typical of either the US or UK population in levels of income and educational attainment. However, as the authors suggest, participants may be reasonably typical of those likely to consider paying for direct-to-consumer genetic testing. The mean age of participants was 46 years, which probably explains the small number who had ever been prescribed drugs for which pharmacogenomic tests were relevant. This small number makes it difficult to determine whether previous concerns raised by the FDA that direct-toconsumer testing might lead to patients stopping certain drugs inappropriately are accurate.

Other concerns expressed by the FDA when deciding to restrict access to direct genetic testing relate to accuracy and interpretation of data returned to consumers. The paper provides detail about the information returned to the participants which supports these concerns about interpretation, while not invalidating the overall conclusions of the study. The data provided on numbers at risk of adverse reactions for abacavir, carbamazepine, azathioprine and flucloxacillin look accurate. However, the information on outcomes of treatment with warfarin, statins and irinotecan could be misleading. Around $80 \%$ of those tested were classified as 'high risk' for warfarin sensitivity and likely to require a 'customized dose'. This seems an inappropriate interpretation of published pharmacogenomic studies which suggest a significantly lower percentage of individuals who are at risk of needing a low dose. ${ }^{3}$ Suggesting that $20 \%$ of participants have a high risk of poor response to certain statins is also contentious. Pharmacogenomic data on statin response are currently limited ${ }^{4}$ and it seems premature to offer advice on this issue. It was also surprising that $50 \%$ of participants were at high risk of irinotecan side-effects. These problems relate partly to the evolving nature of the science. The number of useful tests giving clear pharmacogenomic predictions is still less than the 12 provided to the participants in the study. ${ }^{5}$ In addition, the quality of the interpretation of the warfarin findings offered to participants suggests a need for a better understanding of the underlying literature by the test providers.

In conclusion, this paper illustrates a number of issues around direct-to-consumer pharmacogenomic testing. Though the FDA 'moratorium' has slowed the development of this area, it seems likely that strong public demand for testing worldwide will override the concerns of regulators. It will be important to ensure that information accompanying test results is accurate and that consumers are encouraged to engage with healthcare providers, which the current study suggests the majority are happy to do. This also means that improved pharmacogenomics education for prescribers and other healthcare staff is likely to be important.

\section{REFERENCES}

I https://www.23andme.com

2 Green RC, Farahany NA. Regulation:The FDA is overcautious on consumer genomics. Nature 2014; 505: 286-7.

3 Maitland-van der Zee AH, Daly AK, Kamali F et al. Patients benefit from genetics-guided coumarin anticoagulant therapy. Clin Pharmacol Ther 2014; 96: 15-7. http://dx.doi.org/10.1038/ clpt.2014.44
4 Gelissen IC, McLachlan AJ. The pharmacogenomics of statins. Pharmacol Res 2013. http://dx.doi.org/I0.1016/j.phrs.20I3.12.002

5 Pirmohamed M. Personalized pharmacogenomics: Predicting efficacy and adverse drug reactions. Ann Rev Genom Hum Genet 2014. http://dx.doi.org/I0.I I46/annurev-genom-0904I3-0254I9 